\title{
Analysis of Influences of Shield Tunneling Method on the Existing Upper Road Structures
}

\author{
Wu Liming ${ }^{1}$, Zhou Yi ${ }^{2, *}$, Wang Xiaorong ${ }^{2}$, Wang Zijian ${ }^{2}$ and Piao Lihua ${ }^{3}$ \\ ${ }^{1}$ School of Urban Construction Engineering, Chongqing Business Vocational College, Chongqing, 401520, China \\ ${ }^{2}$ School of Architecture and Engineering, Chongqing University of Science and Technology, Chongqing 40041, China \\ ${ }^{3}$ Bank of Hope, New York, NY11354, United States
}

Received 3 May 2020; Accepted 15 July 2020

\begin{abstract}
It can cause the destruction of the road and even make the road unusable that the uncoordinated deformation of the road and the redistribution of internal forces in the structure When the tunnel passes under the existing road. Nevertheless, existing studies on the influences of tunnel excavation superstructures hardly consider upper pavement load and neglect mutual influences between strata and road structure. A case study on the basis of a rail construction in Jinshuang Interval of Jinyidong Metro in Jinhua City, Zhejiang Province, China was carried out to disclose the influencing laws of shield tunneling method on superstructures more accurately and relieve impacts of underground tunnel construction on road structure. In this case study, a tunnel-soil-road interaction model was constructed using finite element software ANSYS. In addition, variation laws of stress and displacement of road structures caused by tunnel excavation were investigated under lane loading conditions. Influencing laws of tunnel excavation on road structure under an interaction system were disclosed by analyzing the buried depth of the tunnel and the soil parameters of the occupied layer. Results demonstrate that road settlement begins to change significantly and it increases gradually during tunnel advancing. However, road settlement after down-traversing changes slightly, indicating that impacts of tunneling construction on road structure become stable after running through roads. Under collaborative tunnel loads and traffic loads, the maximum settlement of pavement reaches $2.31 \mathrm{~cm}$, which is lower than the widely used control value of $3 \mathrm{~cm}$. The maximum settlement occurs at the upper right position of the tunnel axis. This finding indicates that the influences of tunnel down-traversing on existing road structures are in the safety control range. Settlement of roads increases significantly due to deteriorating soil mass in the occupied soil layer. This finding indicates that the shield tunneling method is inapplicable to soil layers with poor soil quality. Research conclusions can provide theoretical references to practical shield tunneling projects below existing roads.
\end{abstract}

Keywords:tunnel down-traversing, road structures, ANSYS modeling, interaction

\section{Introduction}

With the rapid urbanization process, urban population increases continuously, thereby increasing shortage of land uses. Over ground traffic space has become unable to satisfy traveling demands of people. Therefore, developing underground spaces to relieve traffic pressure in urban areas is necessary. Under this circumstance, metro is highly appreciated in large cities due to its characteristics of large carrying capacity, small land use, high comfort, environmental friendliness, and punctuality. It has become a trunk line in underground traffic transportation in modern large cities. However, tunnel construction surely disturbs surrounding soil and causes stress redistribution of soil, thereby influencing above existing roads and adjacent buildings[ 1 - 4]. When the tunnel runs through above existing roads, it may cause bucking collapse of above road structures when deformation of soil mass is not controlled effectively; it can further cause casualties and property loss. Therefore, influencing laws of tunnel construction on above existing roads should be mastered and specific protective measures should be adopted for road structure security.

*E-muil address: 2y664803746@163.com

ISSN: $1791-2377$ @ 2020 School of Science, IHU. All rights reserved.

doi:10.25103/iestr.134.09
Among existing traditional surface settlement prediction methods in China and foreign countries, the empirical method considers few factors, and model experimental method incurs high costs and great errors [5]. With the development of computer technology, finite element method provides many scholars an effective analytic mean to explore influences of tunnel construction on surface structures. It can often provide more accurate conclusions because it can comprehensively consider dynamic construction process of tunnels, soil characteristics, and architectural structural shapes. However, sensitive influencing factors of tunnel construction on adjacent existing roads should be further studied [6]. Simulation analyses of influences on above existing roads are few, and studies that consider above road loads are limited. Moreover, interaction between soil mass and road structures is ignored. Existing associated studies are mainly simple qualitative analyses, resulting in poor practical performance in construction risk control.

A case study was carried out on the basis of the rail construction project of Jinshuang Interval in Jinyidong Metro, Jinhua City, Zhejiang Province, China to decrease impacts of underground tunnel construction on above road structures and protect life and property safety of the public. A tunnel-soilroad interaction finite element model was constructed through numerical simulation method with considerations to above road loads. Moreover, mechanical properties of road 
structures were investigated to gain relatively accurate and detailed variation laws of stress and displacement of road structures and relieve impacts of underground tunnel construction on road structures.

\section{State of art}

Soil disturbance caused by tunnel excavation directly influence road structures. Disturbance deformation of soil caused by tunnel excavation should be further developed and perfected continuously. Thus far, many scholars have carried out a large number of experimental studies on deformation and deformation laws of soil under influences of tunnel excavation. Some methods have been explored to predict surface settlement deformation, which can be applied to engineering theory and practices, such as empirical formula method and model experimental method. However, these methods mainly provide simple strata settlement prediction. For instance, empirical formula method analyzes abundant practical engineering monitoring data, reviews strata deformation characteristics, and finally obtains strata deformation laws through mathematical statistical analysis. Ağbay [7] pointed out that presupport system of urban shallow-buried tunnel was very important. They introduced a correction factor, which was applicable to ground settlement prediction method of twin-track tunnel, including influences of presupport system. Moreover, a new formula to predict surface deformation caused by construction of twin-track tunnel was proposed. They finally concluded that ground settlement caused by excavation of double-track tunnel was determined by the deformation model of surrounding soil materials in tunnels. Xuan [8] studied the relationship between Peck formula and stepwise mutation model (SMM) theory. On this basis, they proposed a method to determine key SMM parameters and verified its validity by practical engineering projects. They pointed out that Peck formula could be viewed as a simplification of SMM under relatively deep excavation conditions, and the proposed method had very good application effects in practical projects. On the basis of Peck formula and on site testing data, Lu [9] studied the prediction formula of surface deformation caused by curved shield tunneling. They found that the width coefficient of settlement and loss rate of formation showed a linear increase with the increase in buried depth of tunnel. Amir [10] calculated stratum settlement caused by excavation of metro tunnel through empirical formula method, analytical method, and numerical method. They compared the analysis results with practical engineering data from instruments to determine uncertainty of each calculation model. According to research results, the calculated results of the numerical model agreed highly with practical data. Ding [11] believed that calculating stratum settlement caused by tunneling construction below adjacent buildings was unreasonable when using Gaussian curve or Peck curve. They also proposed that stratum settlement presented skewed distribution curve and normal distribution curve features when the tunnel was in and out of the disturbance range. Moreover, the corresponding formulas and parameters were proposed. According to research results, the proposed formula could predict stratum settlement deformation reasonably. These studies on stratum deformation prediction on the basis of empirical formula method hypothesize that soil is a homogeneous medium, predict stratum deformation through calculation formula with limited parameters, and analyze deformation of soil under influences of different factors as well as deformation characteristics. However, surrounding soil media of tunnel excavation are often uneven in practical engineering projects, thereby resulting in large error between prediction results of the empirical formula and the measured results. As a result, the empirical formula method cannot reflect influences of tunnel construction truly. Soil should be considered a homogeneous medium and deformation laws of soil should be studied under influences of tunnel excavation to decrease errors and reflect influences of tunnel excavation on soil more comprehensively.

Soil disturbance caused by tunnel excavation is a complicated process, which is influenced by many factors. To consider influences of soil as a homogeneous medium and reflect actual influences of tunnel excavation on soil, many scholars introduced model experimental method to predict ground settlement. For instance, Peng [12] carried out a unique centrifugal model experiment on ground settlement caused by tunnel construction in loess areas. Subsequently, the settlement of foundation was calculated using the extensively applied method, and the results were compared with measured results in centrifugal model experiment. On this basis, a new method was proposed to evaluate settlement of tunnel construction in loess areas with considerations to special engineering characteristics of loess. According to research results, remarkable differences were found between settlement curves of calculation and measurement. In shallow-buried strata, the settlement curve of the tunnel center line was gentler than that of the centrifugal model experiment. The maximum settlement S-max was relatively low, and great settlement was found far away from the center line. Opposite phenomenon was observed at strata close to the tunnel top. Additional settlement was found in shallow strata due to large porous structure and high compressibility of loess, thereby resulting in such differences. Ding [13] pointed out that rectangular shield tunneling could increase effective use of area and decrease influences of surrounding soil compared with round shield tunneling. Synchronous grouting process was simulated through an integrated simulation test by using large shield tunneling machine. The result showed that slip casting ratio and position of grouting hole were key parameters to synchronous grouting in a rectangular tunnel. Jusoh [14] assessed displacement of surrounding surface of the tunnel, considering the characteristics of segment joints. To assess the influences of segment joints on the overall response of tunnels, a simplified double-slit model and a segment joint model in the all-earth tunnel model were constructed to analyze influences of tunnel construction on surface deformation or settling tank as well as interaction of soil, tunnel, segment joint, and circular seams. Results demonstrated that the influences of different segment joints on the surface were different. In summary, model experimental method can reflect field situations and construction process more truly than empirical formula method, and the results are more intuitive. However, model experimental method incurs high cost, and the results are often sensitive to selection of materials. Thus, large errors are found between prediction results and measured results in practical engineering projects.

Although empirical formula method and model experimental method are easy to operate, they cannot consider factors comprehensively. In practical situations, extremely few soil conforms to empirical formula method and model experimental method, resulting in some limitations of their applications. Tunnel constructions under complicated geological conditions, complicated construction technologies, or complicated tunnel shapes very easily cause large errors 
between prediction results and measured soil deformation in practical field engineering. Thus far, development of numerical simulation technology provides many scholars more convenient and beneficial means to study the influences of tunnel excavation on soil. Numerical simulation method [15] breaks limitations of empirical formula method and model experimental method, as well as classical elasticplastic theory. Ground settlement prediction based on numerical simulation method can consider various factors that might influence settlement. Thus, the results obtained are relatively close to practical engineering projects. For instance, Marto [16] introduced influences of different relative densities of sandy soils on stratum settlement caused by tunnel excavation by studying the parameters of finite element modeling. They found that increasing relative density of sandy soils could decrease influences of tunnel on surface effectively. Eskandari [17] studied stratum response caused by earth-pressure balance (EPB) stress at the triple tunnel intersection of urban railways in Meshed of Iran through ABAQUS and found that influences of tunnel construction sequence on settlement of working surface varied significantly. Moreover, stratum settlement was negatively related with the EPB stress on tunnel section. Aicha [18] constructed a 3D spatial model of PLAXIS and analyzed causes of engineering collapses from three aspects, namely, surface settlement, vertical displacement, and stress of tunnel floor as well as deformation of surrounding supporting soil. Novozhenin [19] simulated stratum settlement changes caused by tunnel construction in complicated soil environments in St. Petersburg through a new technology for escalator tunnel construction by using an EPB advancing machine. Reliability of the proposed technology was proven by determining the maximum settlement point, size of subsider, maximum settlement value, and displacement and strain distribution in subsider. Salvatore [20] carried out a 3D numerical analysis on the tunnel-soil-structure interaction by using a simple elastic-plastic soil constitutive model. The findings reflected major excavation features influencing induced settlement, including advanced and front pressures of tunnel, shield design, and grouting hardening. To inhibit increase in stratum settlement with time, Zhang [21] established a surface settlement model through 3D calculation software FLAC. A calculation formula was deduced and applied to 3D spatial-temporal prediction of double-track tunnel settlement during construction, considering influences of time on surface settlement. Although numerical simulation method is applied widely to predict stratum deformation and road settlement caused by tunnel excavation [22], these studies only investigated influences on above existing roads superficially. Moreover, these studies were mainly based on no-load and hardly consider the upper loads. Research contents were mainly pure qualitative analysis, and most studies applied the two-stage analytical method. They applied an enforced displacement to road structure, or the constructed model ignored mutual influences between stratum and road structure. Relevant conclusions cannot reflect the influencing laws of shield tunneling method on the above road structure accurately.

To address shortages of existing studies, a finite element model of tunnel-soil-road structure was constructed through numerical simulation method on the basis of a case study of rail construction in the Jinshuang Interval of Jinyidong Metro in Jinhua City, Zhejiang Province, China. Influences of tunnel excavation on road structure were investigated under effects of traffic loads. Variation laws of displacement and internal forces of road structures caused by tunnel excavation were investigated from perspectives of buried depth of tunnels and occupied soil parameters. On this basis, relevant conclusions could be drawn.

The remainder of this study is organized as follows. Section 3 provides a brief introduction of the project study. Section 4 describes the finite element modeling method and parameter determination. Section 5 analyzes different finite element model results under different buried depths of the tunnel and soil parameters in the occupied layers. Section 6 summarizes relevant conclusions.

\section{Methodology}

\subsection{Related background}

In this study, the rail construction in the Jinshuang Interval of Jinyidong Metro in Jinhua City, Zhejiang Province, China was chosen as the project study. The left line of the Jinshuang Interval is $2016.919 \mathrm{~m}$ (1681 rings) long, and the right line is $2015.723 \mathrm{~m}$ (1680 rings) long. The minimum radius of the curve is $600 \mathrm{~m}$ and, the soil coverage is $9.2-23.7 \mathrm{~m}$ in the interval. Three connection channels are set in this interval. The affected buildings include Jinlan Community, Baile Hotel, Zhejiang Huayue Real Estate Co., Ltd, and 6\#-7\# concrete buildings of Wenrong Building. The connection runs from the Jiefang West road, Jinhua River, the south foundation of the Shuanglong Bridge, and finally to the Shuangxi West Road Station. The section down-traversing the Jiefang West Road, which is an urban trunk road, was chosen as the study area. The international empirical value (3 $\mathrm{cm}$ ), which is widely accepted to road settlement caused by tunnel down-traversing main trunk road, was used as the control value. The strata within the geological prospecting depth in the study area were plain fill, clay, silt, round gravels, and weathered rocks from upper to bottom section. The distribution laws are shown in Fig. 1. Shield tunneling method was applied in this project. During excavation, the shield tunneling machine advanced gradually by using the front cutting ring, and the tail was responsible for assembly of duct pieces. To prevent excessive settlement of soil, grouting filling of spaces between the shield tail and duct pieces is needed during installation. The designed inner diameter and outer diameter of the supporting duct pieces are 5.4 and $6 \mathrm{~m}$, respectively. The working principle of the shield tunneling machine is shown in Fig.2. Streets in the Jinshuang Interval of Jinyidong Metro in Jinhua City, Zhejiang Province, China were chosen as the numerical simulation research objects, and the tunnel-soil-road interaction model was applied.

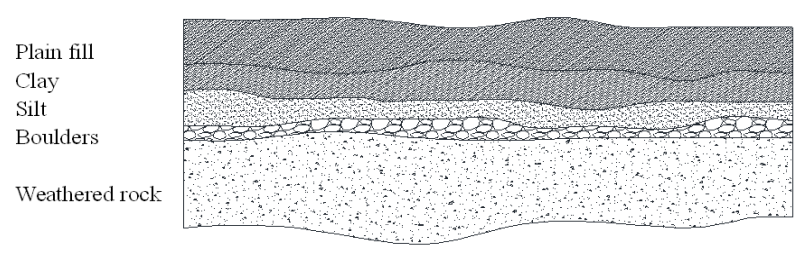

Fig. 1. Distribution laws within the geological prospecting depth in the Jinshuang Interval

\subsection{Basic hypotheses}

Drucker-Prager (DP) model is applicable to constitutive model of geotechnical materials. Thus, the DP yield criteria are approximate to Mahr-Coulomb criteria to amend Von Mises yield criteria. Moreover, DP elastic-plastic constitutive model was chosen to construct models of strata and road 
Wu Liming, Zhou Yi, Wang Xiaorong, Wang Zijian and Piao Lihua/

Journal of Engineering Science and Technology Review 13 (4) (2020) 102 - 109

structures due to its good computational effect, quick convergence, and stable calculation.

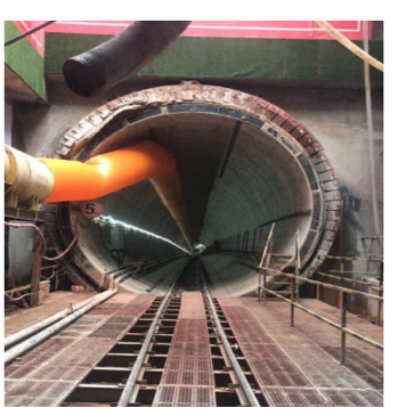

(a)

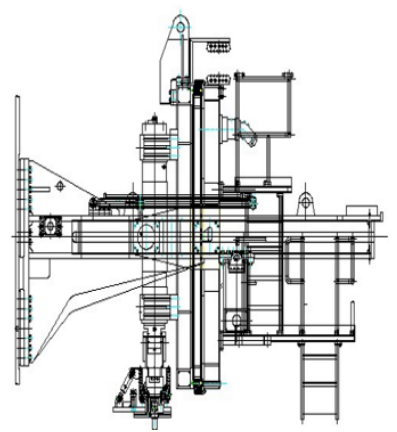

(b)
Fig. 2. Working principle of shield tunneling machine (a) Ventilation system in the tunnel and (b) Installation of duct pieces

Basic hypotheses of the model include the following:

(1) Soil layers in the computational range are homogeneous and isotropic successive elastic-plastic soil. The grouting layer, duct pieces, and road structures are linear elastomers.

(2) During shield tunneling process, loads produced on the excavation surface are uniformly distributed round loads.

(3) At finite element analysis, effects of groundwater and special soil are ignored due to the relatively complicated conditions in underground soil layers.

\subsection{Construction of the computational model}

The tunnel excavation section is simplified into a round shape with a radius of $3.2 \mathrm{~m}$. The buried depth of the tunnel is $12 \mathrm{~m}$. The sphere of influence of the tunnel construction is generally five times the radius of the tunnel. Thus, the soil mass dimension in the computational model is $100 \mathrm{~m}$ (length) $\times 60$ $\mathrm{m}$ (width) $\times 41 \mathrm{~m}$ (height). The tunnel-soil-road interaction model and meshing program are shown in Fig. 3. The complete model has 58,069 units and 59,489 nodes. The buried depth of road and width of urban streets are 0.4 and 24 $\mathrm{m}$, respectively. The mesh generation of lining segment is shown in Fig.4.

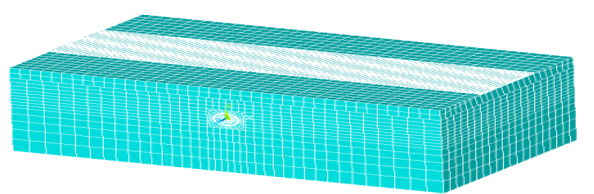

Fig. 3. Tunnel-soil-road interaction model and meshing scheme

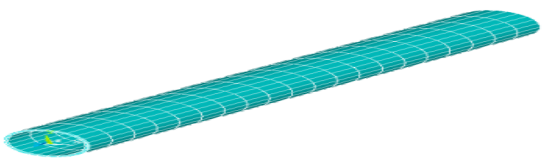

Fig. 4. Meshing scheme of lining ducts

\subsection{Material parameters and unity types}

The constitutive model of soil applied the DP yield criteria and eight-node solid unit. The parameters of soil and lining segment are listed in Table 1. In rock and soil mechanics, the common yield criteria are DP yield criteria, which generally apply DP materials. In finite element analysis of rock and earth masses, DP materials can describe the mechanical properties of geotechnical materials effectively, and results are often more accurate.

DP yield conditions are obtained as follows:

$\alpha I_{1}+\sqrt{J_{2}}=K$

where $I_{1}$ is the first invariant of stress: $I_{1}=\sigma_{x}+\sigma_{y}+\sigma_{z} . \quad J_{2}$ is the second invariant of stress deviation: $J_{2}=\frac{1}{6}\left[\left(\sigma_{x}-\sigma_{y}\right)^{2}+\left(\sigma_{y}-\sigma_{z}\right)^{2}+\left(\sigma_{z}-\sigma_{x}\right)^{2}\right]+\tau_{y z}^{2}+\alpha \quad \alpha \quad \alpha$ and $K$ are constants related to material properties.

Table. 1. Material parameters of soil mass and lining

\begin{tabular}{|c|c|c|c|c|c|c|}
\hline Type & $\begin{array}{l}\text { Thickness } \\
\text { (m) }\end{array}$ & $\begin{array}{l}\text { Density } \\
\left(\mathrm{kg} / \mathrm{m}^{3}\right)\end{array}$ & $\begin{array}{c}\text { Elasticity modulus } \\
\text { (MPa) }\end{array}$ & Poisson ratios & $\begin{array}{c}\text { Cohesion } \\
\text { (kPa) }\end{array}$ & $\begin{array}{c}\text { Internal friction } \\
\text { angle } \\
\left({ }^{\circ}\right)\end{array}$ \\
\hline Plain fill & 5.1 & 1800 & 4.94 & 0.38 & 11 & 13.1 \\
\hline Clay & 4.9 & 1850 & 5 & 0.36 & 19.7 & 19 \\
\hline Silt & 16 & 1980 & 20.6 & 0.31 & 33 & 25.7 \\
\hline Weathered rocks & 15 & 2230 & 500 & 0.27 & 60 & 34.8 \\
\hline Lining ducts & 0.35 & 2500 & 35000 & 0.18 & & \\
\hline
\end{tabular}

The road structural layer applies the eight-node solid units. The road structure and soil are connected by contact units, and frictional effect is considered. Building contact pairs between soil and road structure solves the deformation coordination problem between the soil and road structure effectively. Therefore, stresses in the joint and deformations are successive, and the calculation accuracy of the model is relatively high. The contact pairs are shown in Fig.5. To simplify the model, the pavement structure of above existing road is asphalt concrete, and the foundation is composed of lime-fly ash-stabilized aggregate layer and lime-ash soil layer. Material parameters of road structure are listed in Table 2.

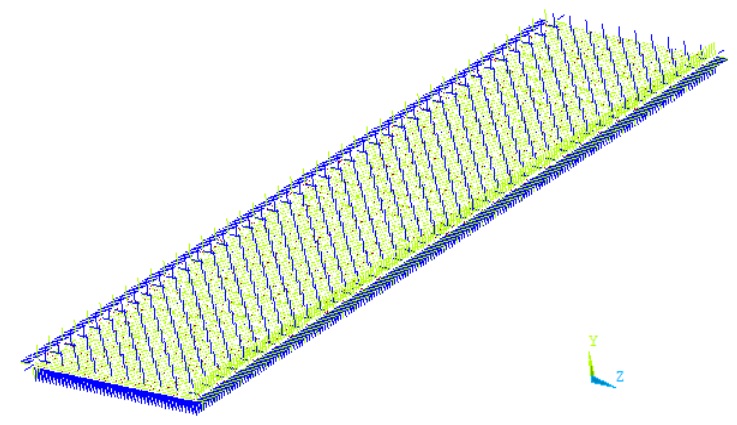

Fig. 5. Contact pair 
Wu Liming, Zhou Yi, Wang Xiaorong, Wang Zijian and Piao Lihua/

Journal of Engineering Science and Technology Review 13 (4) (2020) 102 - 109

Table 2. Material parameters of road structures

\begin{tabular}{|c|c|c|c|c|}
\hline Type & $\begin{array}{c}\text { Thickness } \\
\text { (cm) }\end{array}$ & $\begin{array}{l}\text { Density } \\
(\mathrm{kg} / \mathrm{m} 3)\end{array}$ & $\begin{array}{c}\text { Elasticity modulus } \\
\text { (MPa) }\end{array}$ & Poisson ratios \\
\hline Asphalt concrete pavement & 12 & 2450 & 1430 & 0.35 \\
\hline Lime-fly ash stabilized aggregate layer & 30 & 2300 & 1550 & 0.26 \\
\hline Lime-ash soil layer & 30 & 2200 & 710 & 0.35 \\
\hline Earth base & 50 & 2180 & 48 & 0.37 \\
\hline
\end{tabular}

\subsection{Loads and boundary conditions}

(1) Loads

In the constructed model, lane loads were applied when traffic loads were considered in urban roads, and the remaining loads only considered influences of gravitational field. In accordance with statistics on surface traffic flows, traffic loads were converted into $20 \mathrm{kN} / \mathrm{m} 2$ uniformly distributed loads and then applied onto the pavement [23]. The advancing pressure on the excavation surface was $0.3 \mathrm{MPa}$, and the grouting pressure at shield tail was $0.15 \mathrm{MPa}$.

\section{(2) Boundary constraints}

The model considered displacement boundaries. The parallel direction of the tunnel, direction perpendicular to the tunnel, and vertical directions were set to $\mathrm{Z}$-axis, $\mathrm{X}$-axis, and $\mathrm{Y}$-axis, respectively.

At the bottom of the numerical model, displacements along $\mathrm{X}, \mathrm{Y}$, and $\mathrm{Z}$ directions were restricted. Normal displacements were restricted on four side surfaces. In other words, horizontal displacement was fixed and vertical displacement was free. The top surface of the model used a free boundary.

\section{Results analysis and Discussion}

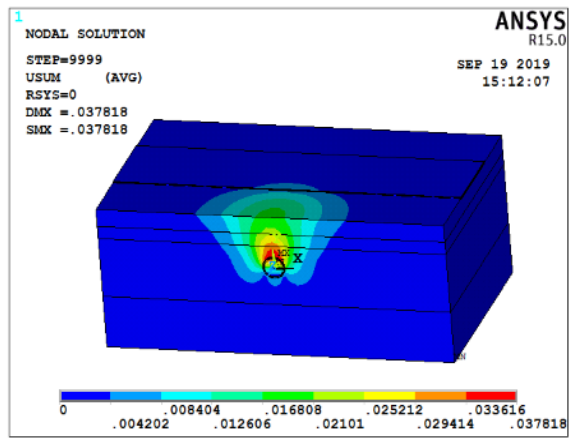

(a) $\mathrm{Z}=12$

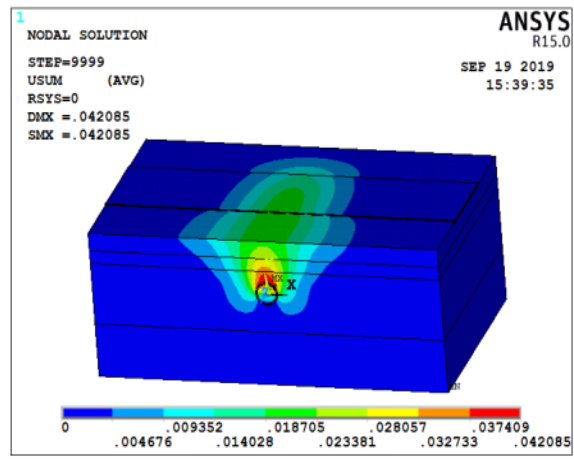

(c) $\mathrm{Z}=42$
4.1 Effects of excavation process on road structure

Shield tunneling is a continuously advancing process. Since the beginning of construction, three synchronous procedures, namely, excavation face, duct assembly, and grouting at shield tail were implemented until the tunnel excavation was completed. In the numerical simulation, the continuously advancing process was studied as a discontinuous excavation process. In other words, dynamic excavation of tunnel was realized by related units of stepwise "generation" and "killing." Vertical displacement prior to tunnel excavation was used as the initial value, and the initial displacement after extraction was subtracted from the vertical displacement to obtain the displacement caused by tunnel extraction. After 20 extraction steps, the shield tunneling ran through the model, and the advancing distance of each step was $3 \mathrm{~m}$.

\subsubsection{Displacement of road structures in the excavation process}

To analyze the complete excavation process, the shield tunneling process was reflected by excavation steps. The review of numerical calculation results revealed the tunnelsoil-road interaction model results when the advancing distance was 12, 30, 42, and $60 \mathrm{~m}$, as shown in Fig.6. As the tunneling process continues, the subsider above the tunnel expanded continuously and showed a V-shaped structure in the front view. The maximum settlement was at the upper right position of tunnel, and it was lower than the universal control value of $3 \mathrm{~cm}$.

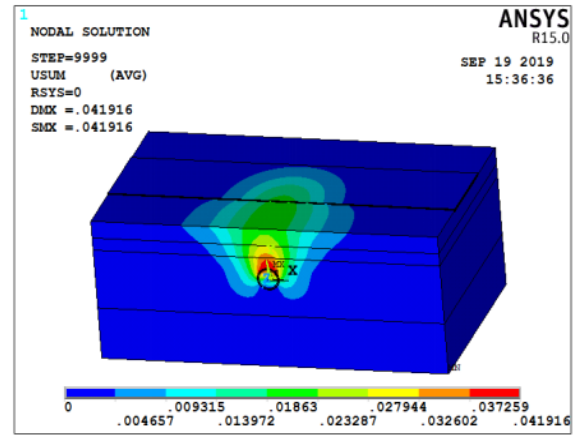

(b) $\quad \mathrm{Z}=30$

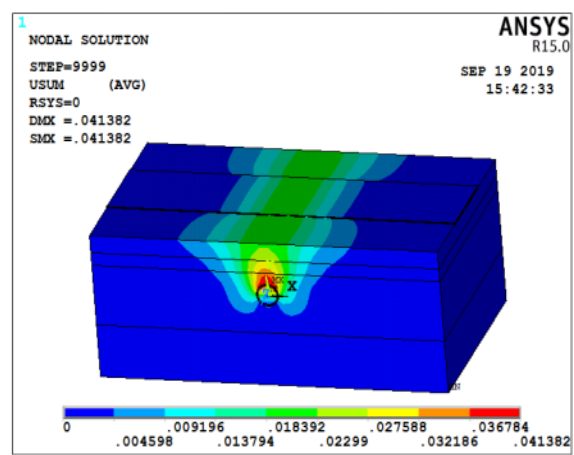

(d) $\quad Z=60$

Fig. 6. Displacement of interaction model in the excavation process 
Wu Liming, Zhou Yi, Wang Xiaorong, Wang Zijian and Piao Lihua/

Journal of Engineering Science and Technology Review 13 (4) (2020) 102 - 109

Eight settlement characteristic points along the $\mathrm{X}$-axis of the pavement central line were chosen as the research objects. The variation laws of settlement could be gained by subtracting the initial displacement from the vertical displacement in the tunneling process (Figs.7 and 8). After tunnel excavation, evident settlement of road structure was found close to the tunnel axial line. The soil above the tunnel was unloaded, and the settlement of road structure began to change greatly due to tunnel construction. The settlement became stable after the tunnel ran through the above road. Under collaborative effects of tunnel and traffic loads, the maximum road settlement reached $2.31 \mathrm{~cm}$, which was lower than the universal control value of $3 \mathrm{~cm}$. The maximum road settlement was at the upper right position of the tunnel axis

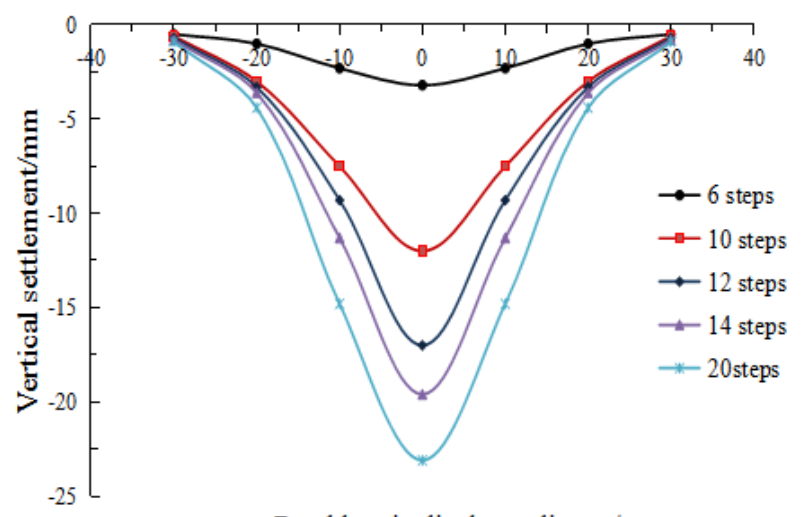

Road longitudinal coordinates $/ \mathrm{m}$

Fig. 7. Vertical displacement on the pavement midline during tunneling

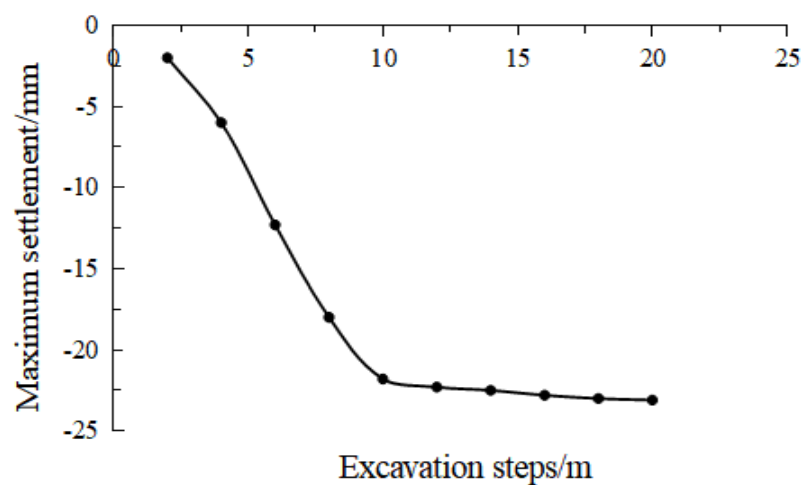

Fig. 8. Maximum vertical displacement of pavement during tunnel

\subsubsection{Stress on the upper road structure in the excavation process}

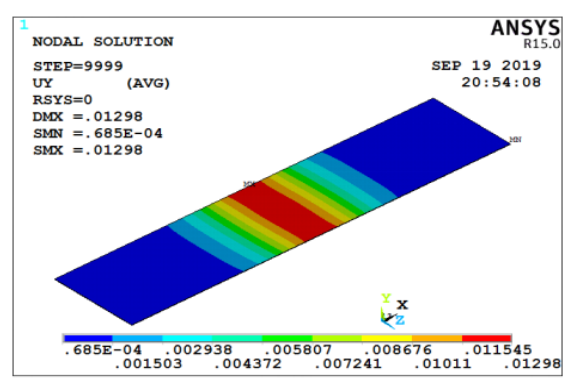

(a)Vertical displacement of silt
Changes of stress on the road structure under collaborative effects of tunnel and lane loads are shown in Fig.9. With the progresses in the tunneling process, stress on the upper road structure increased gradually, but it became stable after running through the road. When the tunnel ran through the road, the maximum stress was detected at the structure below the road center (Fig.10). This situation might cause adverse impacts on stress state in the road center area.

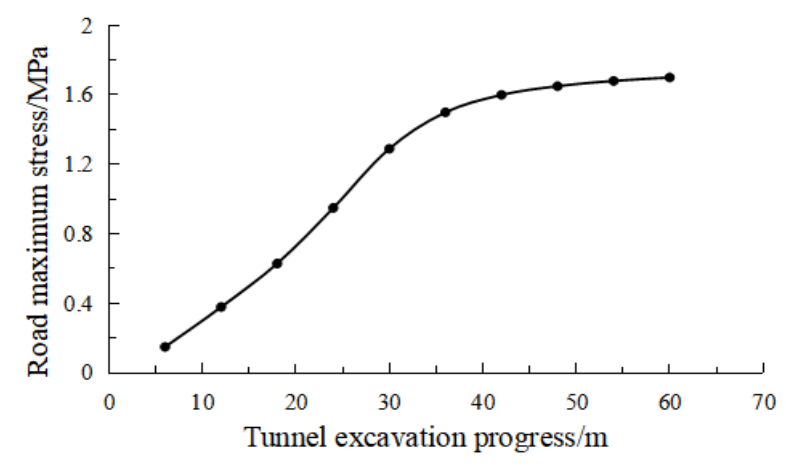

Fig. 9. Maximum stress on the pavement in the tunneling process

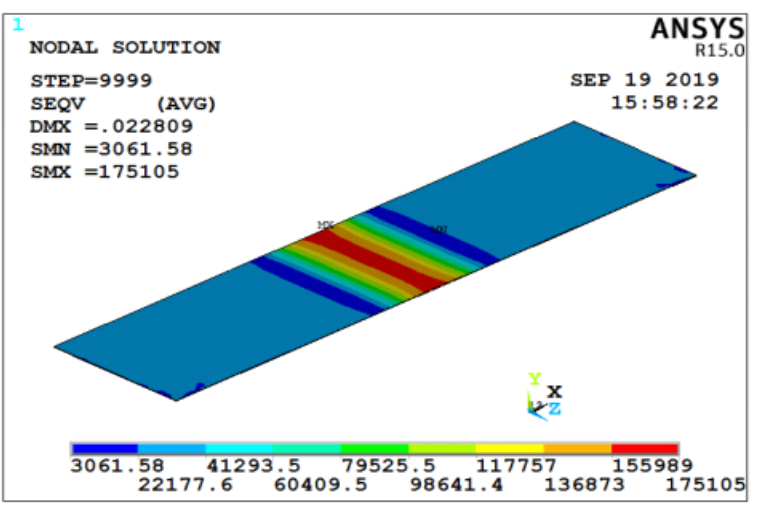

Fig. 10. Maximum stress on the pavement after tunneling

\subsection{Effects of different soil layer parameters}

Four different types of soils were chosen in the overall model to study the influences of soil properties on road structural deformation caused by tunnel excavation. Soil parameters of the occupied soil layers are shown in Table 3. Variations of road deformation with soil properties during tunneling were calculated by developing a tunnel-soil-road interaction model. Results are shown in Fig. 11.

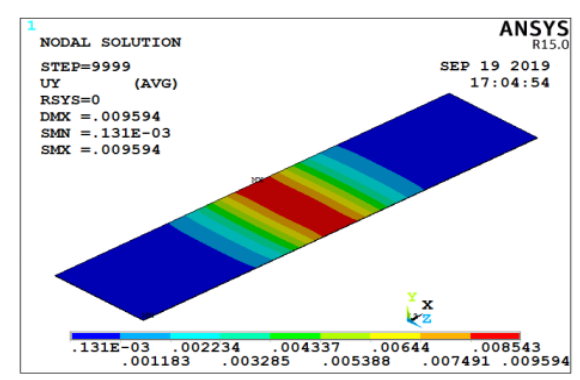

(b) Vertical displacement of medium-coarse sand 


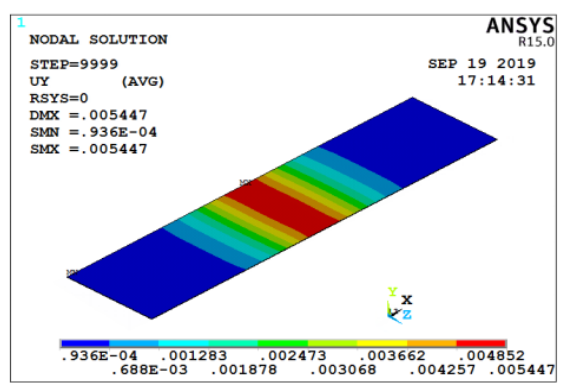

(c) Vertical displacement of gravel

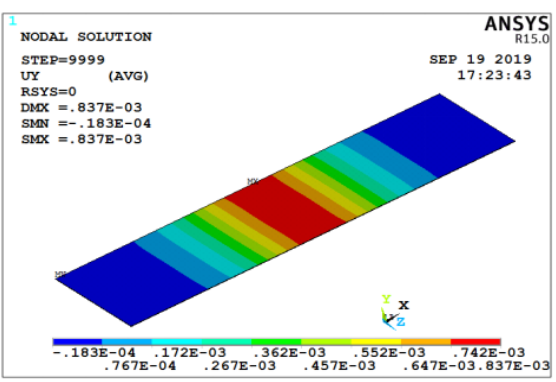

(d) Vertical displacement of weathered rocks
Fig. 11. Variation laws of pavement under different soil properties

Fig.11 shows that when the occupied soil layer was silt, the maximum vertical displacement of pavement was $1.29 \mathrm{~cm}$. When the occupied soil layer included weathered rocks, tunnel excavation influenced the road structure slightly.
Hence, the settlement quantity of road structures caused by tunnel excavation increased significantly when the soil deteriorates.

Table 3. Soil properties in the occupied soil layer

\begin{tabular}{|c|c|c|c|c|c|}
\hline Rocks & $\begin{array}{c}\text { Density } \\
\mathrm{kg} / \mathrm{m} 3\end{array}$ & $\begin{array}{c}\text { Elasticity modulus } \\
\text { (MPa) }\end{array}$ & Poisson ratios & $\begin{array}{c}\text { Cohesion } \\
\text { (kPa) }\end{array}$ & $\begin{array}{c}\text { Internal frictional } \\
\text { angle } \\
\left({ }^{\circ}\right)\end{array}$ \\
\hline Silts & 1980 & 30 & 0.35 & 33 & 25.7 \\
\hline $\begin{array}{l}\text { Medium-coarse } \\
\text { sands }\end{array}$ & 2000 & 44 & 0.31 & 35 & 26.3 \\
\hline Gravels & 2150 & 80 & 0.29 & 44 & 33 \\
\hline Weathered rocks & 2200 & 500 & 0.27 & 60 & 38.8 \\
\hline
\end{tabular}

\section{Conclusions}

A case study on the basis of the Jinshuang Interval of Jinyidong Metro in Jinhua City, Zhejiang Province, China was carried out to improve traditional strata prediction and obtain accurate and detailed stress and deformation laws of road structures. A tunnel-soil-road interaction finite element model was constructed using numerical simulation method. Under traffic loads, displacement and stress variation laws of road structures caused by tunnel extraction were investigated from the buried depth and soil parameters of the occupied soil layers. Some major conclusions could be drawn, as follows:

(1) Shield tunneling method causes evident uneven settlement of the upper existing road structure. Excavation depth influences not only the pavement settlement, but also the shape and size of the settling tank. As the tunneling process continues, the settling tank above the tunnel expands gradually and presents a $\mathrm{V}$ shape in the front view. The maximum settlement is at the upper right position of the tunnel.

(2) Displacement of shield tunneling excavation mainly occurs on soil surrounding the tunnel. The tunnel top develops a relatively large longitudinal displacement due to influences of the above soil and road loads. As the tunneling process continues, the road settlement and maximum stress increase significantly, but they become stable gradually after tunneling.

(3) Influences of soil properties of the occupied soil layers are analyzed. The results show that soil properties of the occupied soil layers influence the existing road deformations significantly. The vertical road settlement increases greatly when soil is deteriorated. This finding indicates that shield tunneling method is inapplicable to environment with poor soils.

Road settlement caused by shield tunneling below existing roads is analyzed by numerical simulation method. A tunnel-soil-road interaction finite element model, which can obtain more accurate and detailed variation laws of stress and deformation of road structures, is constructed. However, this study only focuses on horizontal road structures and static impacts of traffic loads. Future studies shall be further perfected by considering dynamic influences.

\section{Acknowledgements}

The authors are grateful for the support provided by open funding project of Key Laboratory of Ministry of Education, Port\& Waterway Engineering (SLK2017B03),Planned project of Infrastructure and Leading Researches of Chongqing City (cstc2017jcyjAX0189),Scientific and Technological Research Project of Education Committee of Chongqing City (KJQN201804010), Fund Project of Technological Colleges of Chongqing City (CK2016B09) and Key Scientific Research Projects of Chongqing Vocational College of Commerce and Industry in 2019 (NDZD2019-01)

This is an Open Access article distributed under the terms of the Creative Commons Attribution License

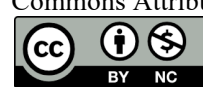

\section{References}

1. Shi, C. H., Cao, C. Y., Lei, M. F., "An analysis of the ground deformation caused by shield tunnel construction combining an elastic half-space model and stochastic medium theory". KSCE Journal of Civil Engineering, 21(5), 2017, pp. 1933-1944.
2. Shi, J. W., Zhang, X., Chen, L., "Numerical Investigation of Pipeline Responses to Tunneling-Induced Ground Settlements in Clay". Soil Mechanics and Foundation Engineering, 54(5), 2017, pp. 303-309. 
Wu Liming, Zhou Yi, Wang Xiaorong, Wang Zijian and Piao Lihua/

Journal of Engineering Science and Technology Review 13 (4) (2020) 102 - 109

3. Li, X. Z., Wang, G. F., "In-Situ Testing Study on Supporting System Stability of Phyllite Tunnel". Electronic Journal of Geotechnical Engineering, (19), 2014, pp. 341-354.

4. Mao, X. X., Chen, B. G., Jiang, C. X., She, M. K., "Research on the Influence Law of Shield Under passing Near Highway Tunnel". Highway, 63 (04), 2018, pp. 258-263.

5. Zhang, W., "Research on Stratum Deformation Law and Control Method of Metro Tunnel Crossing Existing Municipal Road". Master thesis of Shandong University, China, 2017, pp. 23-28.

6. Camós, C., Molins, C., Arnau, O., "Case Study of Damage on Masonry Buildings Produced by Tunneling Induced Settlements". International Journal of Architectural Heritage, 8(4), 2014, pp. 602 625 .

7. Ağbay, E., Topal, T., "Evaluation of twin tunnel-induced surface ground deformation by empirical and numerical analyses (NATM part of Eurasia tunnel, Turkey)". Computers and Geotechnics, 119, 2020, pp.103373.

8. Xuan, R. Z., "Determination of Main Influence Angle of Ground Settlement Caused by Xi'an Metro Tunnel Construction". Advanced Materials Research, 327, 2014, pp. 1065-1069.

9. Lu, L. H., Sun, J. C., Zhou, G. F., "Research on the Surface Deformation Prediction for Curved Shield Construction in Clay Stratum”. Journal of Railway Engineering Society, 35(05), 2018, pp. 99-105.

10. Amir, K., Hamed, A., Raheb, B., "Model uncertainty of various settlement estimation methods in shallow tunnels excavation; case study: Qom subway tunnel". Journal of African Earth Ences, 134(10), 2017, pp. 658-664.

11. Ding, Z., Wei, X. J., Wei, G., "Prediction methods on tunnelexcavation induced surface settlement around adjacent building". Geomechanics and Engineering, 12(2), 2017, pp. 185-195.

12. Peng, H., Zhang, L. J., Zhang, L. Y., Chen, R., Yang, W. J., “A New Method based on Centrifuge Model Test for Evaluating Ground Settlement Induced by Tunneling”. KSCE Journal of Civil Engineering, 23(6), 2019, pp. 2426-2436.

13. Ding, W. Q., Duan, C., Zhu, Y. H., "The behavior of synchronous grouting in a quasi-rectangular shield tunnel based on a large visualized model test". Tunnelling and Underground Space Technology, 83(1), 2019, pp. 409-424.
14. Jusoh, S. N., Mohamad, H., Marto, A., "Assessment on Segment Joint to Improve Soil-Tunnel Interaction". Matec Web of Conferences, 250(04), 2018, pp.01005.

15. Yuan, H. L., Zhang,Y., Cui Y. W., "Settlement Analysis of Grouting Scheme for Undercut Tunnel Under Municipal Road". Engineering Construction, 51 (01), 2019, pp. 69-73 + 86.

16. Marto, A., Sohaei, H., Hajihassani, M., "Effects of tunnel depth and relative density of sand on surface settlement induced by tunneling". Electronic Journal of Geotechnical Engineering, 20(3), 2015, pp.1045-1052.

17. Eskandari, F., Goharrizi, K.G., Hooti, A., "The impact of EPB pressure on surface settlement and face displacement in intersection of triple tunnels at Mashhad metro". Geomechanics and Engineering, 15(2), 2018, pp.769-774.

18. Aicha, B., Toufik, K., "Numerical modeling of the pathological case of a damaged tunnel application to Djebel El-Ouahch tunnel (east - west highway)". Asian Journal of Civil Engineering, 19(8), 2018, pp. 913-925.

19. Novozhenin, S. U., Vystrchil, M. G., "New Method of Surface Settlement Prediction for Saint-petersburg Metro Escalator Tunnels Excavated by EPB TBM". Procedia Engineering, 150, 2016, pp. 2266-2271

20. Salvatore, M., Armando, D. L., "Predicted and observed settlements induced by the mechanized tunnel excavation of metro line $\mathrm{C}$ near $\mathrm{S}$. Giovanni station in Rome". Tunnelling and Underground Space Technology, 86(APR.), 2019, pp. 236-246.

21. Zhang, Q. J., Wu, K., Cui, S. S., Yu, Y. L., Zhang, Z., Zhao, J. H., "Surface Settlement Induced by Subway Tunnel Construction Based on Modified Peck Formula". Geotechnical and Geological Engineering, 37(4), 2019, pp. 2823-2835.

22. Dong, Q., Wan, H. Y., Kong, F. L., Zhao, B. Y., "The 3D Numerical Simulation and Settlement Control Study for City Tunnel Crossing High Fill Geological Area of Gravelly Soils". Applied Mechanics and Materials, 2156, 2013, p. 1235-1242.

23. Wang, Z. C., Wang, C., Zhu, X. N., Zhao, D. S,. "Analysis of the influence of shield tunnel construction on adjacent building structures". Chinese Science and Technology Papers,13 (13), 2018, pp. 1488-1493. 